\title{
Editorial: Material and Composition Screening Approaches in Electrocatalysis and Battery Research
}

\author{
Thomas Kadyk ${ }^{1,2}$, Jianping Xiao ${ }^{3}$, Hideshi Ooka ${ }^{4}$, Jun Huang ${ }^{5}$ and Kai S. Exner ${ }^{6,7,8 *}$ \\ ${ }^{1}$ Theory and Computation of Energy Materials (IEK-13), Institute of Energy and Climate Research, Forschungszentrum Jülich \\ $\mathrm{GmbH}$, Jülich, Germany, ${ }^{2}$ Jülich Aachen Research Alliance, JARA Energy, Jülich, Germany, ${ }^{3}$ State Key Laboratory of Catalysis, \\ Dalian Institute of Chemical Physics, Chinese Academy of Sciences, Dalian, China, ${ }^{4}$ Center for Sustainable Resource Science \\ (CSRS), RIKEN, Wako, Japan, Institute of Theoretical Chemistry, Ulm University, Ulm, Germany, ${ }^{6}$ Faculty of Chemistry, \\ Theoretical Inorganic Chemistry, University Duisburg-Essen, Essen, Germany, ${ }^{7}$ Cluster of Excellence RESOLV, Bochum, \\ Germany, ${ }^{8}$ Center for Nanointegration (CENIDE) Duisburg-Essen, Duisburg, Germany
}

Keywords: materials screening, electrocatalysis, solid-state batteries, electrolyte composition, machine learning

\section{Editorial on the Research Topic}

Material and Composition Screening Approaches in Electrocatalysis and Battery Research

In light of the global effort to transform the energy supply away from fossil fuels toward renewables, electrochemical devices have emerged as key technologies to store and convert energy as well as to convert waste products such as $\mathrm{CO}_{2}$ into high-value chemicals. Progress in advancing these technologies (i.e., fuel cells, batteries, electrolyzers, or $\mathrm{CO}_{2}$ conversion cells) hinges on the development and improvement of key materials, especially electrocatalysts and charge storage materials as well as electrolytes and transport materials. The complexity of the structure and composition of the state-of-the-art materials synthesized in recent years has made the search for new materials challenging. Trial-and-error-based screening of new materials is becoming less effective, as

Edited and Reviewd by: Sheng S. Zhang, United States Army Research Laboratory, United States

*Correspondence: Kai S. Exner

kai.exner@uni-due.de

Specialty section:

This article was submitted to Electrochemical Energy Conversion and Storage, a section of the journal Frontiers in Energy Research

Received: 23 April 2021 Accepted: 30 April 2021

Published: 14 May 2021

Citation:

Kadyk T, Xiao J, Ooka H, Huang J and Exner KS (2021) Editorial: Material and Composition Screening Approaches in Electrocatalysis and Battery Research. Front. Energy Res. 9:699376. doi: 10.3389/fenrg.2021.699376 there are too many material combinations, synthesis parameters and processing routes to try experimentally. Hence, effective screening methods to designate experimental targets are required to advance the discovery of next-generation materials in an effective way. The development of advanced screening methods has led scientists on the hunt for the understanding of underlying relationships (e.g., structure-property-performance), and guiding principles (like the Sabatier principle). Additionally, the use of ever-more-powerful computational methods to avoid cumbersome, expensive experiments, as well as the utilization of machine learning and artificial intelligence techniques to re-create in-silico the intuition and experience of an experimenter, leads to a further increase of the efficiency of materials screening methods.

The articles comprised in this special issue highlight a broad range of applications, in which materials and composition screening is used. They range from advanced battery technologies (Dillenz et al.; Tichter et al.; Kowalski et al.), to water splitting (Mukouyama and Nakanishi; Lim et al.; Zeradjanin et al.) and $\mathrm{CO}_{2}$ reduction (Malek et al.; Verma et al.) to general electrocatalysis (Kox et al.; Ooka et al.). The applied methods span the whole spectrum from computational methods like density functional theory (Dillenz et al.; Kowalski et al.; Lim et al.; Verma et al.) and ab-initio molecular dynamics simulations (Kox et al.), continuum modeling (Mukouyama and Nakanishi), artificial intelligence (Malek et al.), as well as the interplay of theory and experiment (Mukouyama and Nakanishi; Tichter et al.).

Apart from the 8 Original Research articles, this special issue contains 2 review articles. Our editors Ooka, Huang and Exner have reviewed the famous Sabatier principle within the context of electrocatalysis. They have highlighted its limitations and challenges, and in doing so, have showed how moving beyond its current thermodynamic framework might lead to next-generation 
electrocatalysts. In the second review, Zeradjanin et al. have reviewed the current understanding of activity and stability trends of oxides for the anodic oxygen evolution reaction (OER). Their review brilliantly highlights that the topic is far from being concluded and ends with remaining fundamental questions and eight suggestions for future research directions.

Lim et al. partially followed one of the suggested directions by tuning the OER activity of transition-metal oxides via the strategic formation of a heterostructure with another transition metal oxide. They screened 11 transition metal oxides on $\mathrm{a} i O_{2}$ substrate using DFT, finding that these heterostructures follow the universal scaling relationship of metal oxides, thereby confirming $\mathrm{RuO}_{2}$ and $\mathrm{IrO}_{2}$ as highly active OER catalysts.

Staying in the field of electrochemical water splitting, Mukouyama and Nakanishi investigated the hydrogen evolution reaction and highlighted the importance of quantifying the surface $\mathrm{pH}$. They developed and demonstrated an effective continuum model, which converts partial differential equations to ordinary differential equations, allowing the surface $\mathrm{pH}$ to be estimated in a computationally efficient way. The resulting model explains measured experimental voltammograms of both the hydrogen evolution as well as the more complex hydrogen peroxide reduction reaction.

Another work focusing on the catalyst-electrolyte interface is presented by Kox et al. Using ab-initio molecular dynamics simulations, they unraveled the effect of water and solvation on the structure and reactivity of $\mathrm{Co}_{3} \mathrm{O}_{4}$ (001) A-type and B-type surface terminations.

Moving to the application in batteries, Tichter et al. have investigated the electro-oxidation of $\mathrm{VO}^{2+}$ on glassy carbon electrodes, as encountered in redox-flow batteries. They performed stationary and rotating linear sweep voltammetry, which they combined with Koutecký-Levich analysis. While the observed concentration dependence of the ordinate intercept in the Koutecký-Levich plots was so far unexplained by the theory, they introduced a concept of finite rate constants leading to a theory that captures mass transport limitations, Butler-Volmer kinetics, and finite heterogenous kinetics simultaneously.

In solid-state lithium ion batteries, Kowalski et al. gave an overview and discussed the role of atomistic modeling in accurately predicting thermodynamic properties of $\mathrm{Li}_{x} \mathrm{FePO}_{4}$ orthophosphates as well as fluorite- and pyrochlore-type zirconates, key materials for energy storage and solid-state ion conduction. Dillenz et al. also used periodic density functional theory calculations to screen the migration of various charge carriers in spinel-type $\mathrm{MgSc}_{2} \mathrm{Se}_{4}$, a potential candidate for solid electrolytes in $\mathrm{Mg}$-ion batteries. Screening the diffusion barriers of different ions in this material allowed disentangling structural and chemical factors in ion mobility. Not only the size and charge of the ion determines its mobility, but also charge redistribution and rehybridization caused by the migration of multivalent ions increase the resulting migration barriers.

Tackling the challenge to find suitable electrocatalysts for $\mathrm{CO}_{2}$ reduction to $\mathrm{CO}$, Verma et al. used computational screening of doped graphene electrodes. After establishing thermodynamically stable electrode materials, the $\mathrm{CO}_{2}$ reduction reaction in alkaline media was studied. It was found that the $\mathrm{CO}_{2}$ electrosorption and associated charge transfer along the decoupled proton and electron transfer mechanism significantly impacts the electrochemical performance, leading to their discovery of metal-doped 3 nitrogen-coordinated graphene as highly active electrodes.

Malek et al. made use of computational and experimental materials data in an artificial intelligence-based material recommendation and screening framework. This framework utilizes high-level technical targets, advanced data extraction, and categorization as well as data analytics and propertymatching algorithms to recommend the most viable materials and reveal correlations that govern catalyst performance. This framework is demonstrated for certain classes of electrocatalyst materials for low or high temperature $\mathrm{CO}_{2}$ reduction.

Overall, we thank all authors for their excellent, broad and multi-facetted contributions that highlight the importance and widespread application of diverse materials screening approaches and show up pathways for future energy materials discovery.

\section{AUTHOR CONTRIBUTIONS}

TK drafted the editorial. All authors discussed, reviewed, and approved the submission of this editorial.

Conflict of Interest: The authors declare that the research was conducted in the absence of any commercial or financial relationships that could be construed as a potential conflict of interest.

Copyright (c) 2021 Kadyk, Xiao, Ooka, Huang and Exner. This is an open-access article distributed under the terms of the Creative Commons Attribution License (CC $B Y)$. The use, distribution or reproduction in other forums is permitted, provided the original author(s) and the copyright owner(s) are credited and that the original publication in this journal is cited, in accordance with accepted academic practice. No use, distribution or reproduction is permitted which does not comply with these terms. 